\title{
Regenerative medicine in China: new advances and hopes
}

\author{
Xiaobing $\mathrm{Fu}^{*}$ \\ Institute of Basic Medical Sciences, Chinese PLA General Hospital, Beijing 100853, China
}

Received September 12, 2018; published online September 26, 2018

Citation: $\quad F u, X$. (2018). Regenerative medicine in China: new advances and hopes. Sci China Life Sci 61, 1135-1136. https://doi.org/10.1007/s11427-018-9394-9

Tissue and organ repair as well as regeneration are global frontiers in the field of stem cell research and regenerative medicine. Regenerative medicine is closely associated with treatments of almost all human diseases, injuries, and aging-related problems. The rapid advancements in stem cell technology, tissue engineering, and physical as well as chemical interventions have resulted in significant progress in tissue and organ repair as well as regeneration. Currently, a single tissue type can be repaired and regenerated through cell transplantation, trophic factors, biodegradable scaffolds, $3 \mathrm{D}$ printing technology, and pharmaceuticals. However, traumatic injuries often involve simultaneous damage to multiple tissue types, which include the skin, musculoskeletal system, vessels, and nerves. The current approaches have failed to enable the synchronized repair and regeneration of multiple tissues, which frequently results in patients with permanent, life-long disabilities. By contrast, lower animals, such as salamanders and zebrafish, can completely regenerate their appendages (e.g., limbs, fins, and tails) or entire organs (e.g., hearts) in situ after amputation. Thus, achieving perfect repair and regeneration of multiple mammalian tissues, despite its difficulties, should be the focus of future research in regenerative medicine.

Based on knowledge of the regenerative processes in lower animals and the current status of regenerative medicine, I propose three main routes to achieve the synchronized repair and regeneration of multiple tissues and the reconstruction of large organs. One route is to induce the differentiation of stem cells into multiple tissues or organs, thus imitating embryonic development. For example, in-

*Corresponding author (email: fuxiaobing@vip.sina.com) duced pluripotent stem cells (iPSCs) have been demonstrated to differentiate into kidney organoids. The second route is to simultaneously create multiple tissues and functional large organs using tissue engineering and 3D printing technologies. The third route is to induce in situ regeneration of multiple tissues and large organs at the injury site through small molecules, biomaterials, and modulation of the injured microenvironment. Among these routes, the in situ regeneration of multiple tissues and large organs is surmised to be the ideal method to restore the structures and functions of tissues and organs after injuries and diseases. The in vivo modulation of cell fates may play a central role in promoting in situ regeneration. There are three strategies to produce functional cells for regenerating multiple tissues: first, by stimulating the activation, proliferation, and differentiation processes of endogenous stem cells from different tissue lineages; second, in vivo dedifferentiation, proliferation, and redifferentiation of diverse terminally differentiated cells; and third, in vivo reprograming or transdifferentiation of resident differentiated cells into lost or dead cells. Studies have shown that in vitro cell fate is controlled by genetic, biological, chemical, and physical tools. The application of these tools to adult stem cells and differentiated cells in vivo is expected to generate new functional cells for in situ regeneration. In addition, changing injured, unfavorable microenvironments into regeneration-permissive microenvironments will facilitate the regenerative processes. We hope that the development of regenerative medicine in China will result in breakthroughs in the regeneration of multiple tissues and large organs.

In this special topic, three research groups have reported on their recent studies regarding the chemical conversion of 
somatic cells, endothelialization in engineering vessels, and microenvironment reconstruction, respectively.
Compliance and ethics The author(s) declare that they have no conflict of interest.

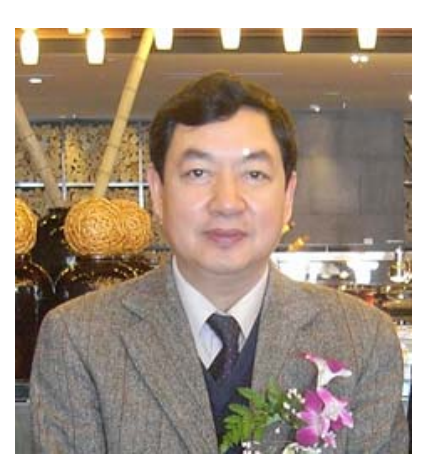

\section{Biographical Sketch}

Dr. Xiaobing Fu is a Professor and Director of the Key Research Laboratory of Wound Repair and Regeneration of PLA, the Director of the Institute of Basic Medical Science, College of Life Sciences, General Hospital of PLA (301 Hospital), Medical College of PLA, and the President of the Chinese Tissue Repair Society and the Chinese Trauma Society. He serves as a member of the World Union of Wound Healing Societies and an editorial board member of international journals such as Wound Repair and Regeneration, International Wound Journal, International Lower Extremity Wound Journal, Advances in Wound Care and Science China Life Sciences. He was elected to the Chinese Academy of Engineering (Division of Medical and Health) in 2009.

Dr. Fu has made significant contributions to the fields of trauma, tissue repair, and regeneration, particularly in the fields of growth factor functions and their effects in regulating wound healing and stem cell biology as well as their application in sweat gland regeneration. His research has been supported in part by the National Basic Research Program of China, National Distinguished Young Scientists, and National Natural Science Foundation of China. He has published more than 600 scientific papers, including approximately 260 papers in international journals. As an editor-in-chief, he has published 25 books, including Regenerative Medicine: Basic Principle and Practices and Modern Traumatology. He has won three first prizes of the National Science and Technology Progress Award (one as the first accomplisher and two as the second accomplisher). He has won numerous international and national prizes for his achievements in the past 30 years. 